\title{
Regulation of the Notch-ATM-abl axis by geranylgeranyl diphosphate synthase inhibition
}

\author{
Sherry S. Agabiti ${ }^{1}$, Jin Li', Willie Dong ${ }^{1}$, Michael M. Poe ${ }^{1}$ and Andrew J. Wiemer $\mathbb{B}^{1,2}$
}

\begin{abstract}
Notch proteins drive oncogenesis of many cancers, most prominently T-cell acute lymphoblastic leukemia (T-ALL). Because geranylgeranylated Rab proteins regulate Notch processing, we hypothesized that inhibition of geranylgeranyl diphosphate synthase (GGDPS) would impair Notch processing and reduce viability of T-ALL cells that express Notch. Here, we show that GGDPS inhibition reduces Notch1 expression and impairs the proliferation of T-ALL cells. GGDPS inhibition also reduces Rab7 membrane association and depletes Notch1 mRNA. GGDPS inhibition increases phosphorylation of histone H2A.X, and inhibitors of ataxia telangiectasia-mutated kinase (ATM) mitigate GGDPS inhibitor-induced apoptosis. GGDPS inhibition also influences c-abl activity downstream of caspases, and inhibitors of these enzymes prevent GGDPS inhibitor-induced apoptosis. Surprisingly, induction of apoptosis by GGDPS inhibition is reduced by co-treatment with $\gamma$-secretase inhibitors. While inhibitors of $\gamma$-secretase deplete one specific form of the Notch1 intracellular domain (NICD), they also increase Notch1 mRNA expression and increase alternate forms of Notch1 protein expression in cells treated with a GGDPS inhibitor. Furthermore, inhibitors of $\mathrm{Y}^{-}$ secretase and ATM increase Notch1 mRNA stability independent of GGDPS inhibition. These results provide a model by which T-ALL cells use Notch1 to avoid DNA-damage-induced apoptosis, and can be overcome by inhibition of GGDPS through effects on Notch1 expression and its subsequent response.
\end{abstract}

\section{Introduction}

T-cell acute lymphoblastic leukemia (T-ALL) is a rare but aggressive T-cell malignancy. T-ALL comprises $\sim 15 \%$ of childhood and $25 \%$ of adult ALL cases ${ }^{1}$. While the 5year survival rate in children $(80 \%)$ has improved significantly due to advances in chemotherapy, survival in primary resistant and relapsed cases is still $\operatorname{low}^{2}$, and many current therapies often cause toxicity in healthy cells.

One common mutation in T-ALL is NOTCH1, which is altered in over $60 \%$ of T-ALL cases ${ }^{3}$. Notch is also mutated in other types of cancer, such as breast, colon, lung, and prostate cancer ${ }^{4}$. However, its role in cancer is context dependent as Notch is oncogenic in some models

\footnotetext{
Correspondence: Andrew J. Wiemer (andrew.wiemer@uconn.edu)

${ }^{1}$ Department of Pharmaceutical Sciences, University of Connecticut, Storrs, CT 06269, USA

${ }^{2}$ Institute for Systems Genomics, University of Connecticut, Storrs, CT, USA Edited by H.-U. Simon
}

but functions as a tumor suppressor in others ${ }^{5}$. Notch signaling is evolutionarily conserved and is required at multiple points in the developmental process, particularly in T-cell development ${ }^{6,7}$. Notch is first processed in the Golgi by furin convertases ${ }^{4}$. It is then transported to the plasma membrane where it encounters its ligand (delta/ serrate/LAG-2) ${ }^{8}$. Ligand binding promotes two cleavage events, one by Adam 10 and the other by $\gamma$-secretase ${ }^{9}$. It has been proposed that Notch activation cannot occur without $\gamma$-secretase processing. Hydrolysis by $\gamma$-secretase processing releases the active form of Notch, the Notch internal domain (NICD), which can translocate to the nucleus, bind to transcription factors, and promote proliferation and differentiation ${ }^{10}$.

Recent findings suggest a role for small GTPases in Notch1 processing. Court et al. showed a requirement of Rab7 and Rab8 in Notch1 signaling ${ }^{11}$, while Doi et al. found that Rap1 is essential in maturation of Adam $10^{12}$. At the same time, we identified selective inhibitors of the 
enzyme geranylgeranyl diphosphate synthase (GGPS1, or GGDPS), which can be used to disrupt the function of geranylgeranylated small GTPases ${ }^{13-19}$. GGDPS is an enzyme in the isoprenoid biosynthesis pathway that produces geranylgeranyl diphosphate (GGPP) whose lipid moiety is post-translationally attached to small GTPases such as Rab and Rap to promote membrane association $^{20,21}$. Thus, we hypothesized that disrupting small GTPase lipidation with a GGDPS inhibitor would affect Notch1 signaling and display beneficial growth inhibition in T-ALL.

Because drugs such as statins and nitrogenous bisphosphonates deplete GGPP and are implicated as cancer therapeutics, it is important to understand the signaling pathways affected by GGPP depletion. Throughout these studies, we use an inhibitor of GGDPS, digeranyl bisphosphonate (DGBP), to directly deplete levels of GGPP. DGBP has a GGDPS $\mathrm{IC}_{50}$ of $200 \mathrm{nM}$ and is well characterized, making it useful to determine how GGPP depletion leads to apoptosis ${ }^{13,14}$. Better understanding of the mechanisms linking GGDPS inhibition to proliferation will be useful as further developments are made regarding the preclinical development of GGDPS inhibitors $^{22-24}$ and geranylgeranyl transferase $\mathrm{I}^{25,26}$ and $\mathrm{II}^{27,28}$ inhibitors. Here, we describe the mechanism by which GGPP depletion alters Notch1 expression and function and induces apoptosis.

\section{Materials and methods}

\section{Supplies and materials}

The cell lines Jurkat, Daudi, and HL-60 were obtained from ATCC (Manassas, VA, USA). Molt-4 cells were obtained from ATCC. K562 cells were obtained from Sigma Aldrich (Saint Louis, MO). RPMI-8226 cells were obtained from Coriell Institute (Camden, NJ). U2OS cells were obtained from ATCC. Cells were cultured in media containing RPMI-1640, 10\% FBS, and Pen/Strep. Loucy cells were obtained from ATCC and cultured in T-cell media (RPMI-1640, 10\% FBS, penicillin/streptomycin, nonessential amino acids, sodium pyruvate, $0.0004 \% 2$ mercaptoethanol, and $10 \mathrm{mM}$ HEPES, pH 7.5). Cells were grown for at least 2 weeks after thawing before experimental use, and replaced with a fresh early-passage stock after no more than 3 months. GGPP, DAPT, and coelenterazine were obtained from Cayman Chemical (Ann Arbor, MI, USA). Z-VAD-FMK was obtained from ApexBio (Houston, TX, USA). Imatinib, nilotinib, and Ku55933 were obtained from LC laboratories (Woburn, MA, USA). FITC-conjugated annexin V was obtained from Biolegend, and propidium iodide was obtained from Fisher.

Retinoblastoma protein (C-2) and c-myc (C-33) antibodies came from Santa Cruz (Santa Cruz, CA, USA). Cleaved caspase 9 (Asp330) (D2D4), p-H2A.X (S139)
(20E3), ATM (D2E2), cleaved Notch1 (V1744) (D3B8) (corresponding to human V1754), and Rab7 (D95F2) antibodies were from Cell Signaling Technologies (Danvers, MA). $\beta$-actin (Poly 6221) and c-abl (8E9) antibodies were obtained from Biolegend (San Diego, CA, USA). Vinculin (hVIN-1) was purchased from Sigma. The antibody for the Notch1 internal domain (bTAN20) was purchased from the Developmental Studies Hybridoma Bank (deposited by ArtavanisTsakonas ${ }^{29}$ ). The antibody for actin (JLA20) was obtained from the Developmental Studies Hybridoma Bank (deposited by Lin J.J. ${ }^{30}$ ).

pCIneoRL-Notch1 3'UTR was purchased from Addgene (plasmid \# 84595) (deposited by Yanan Yang ${ }^{31}$ ).

\section{Apoptosis analysis}

Cells were seeded at 100,000 cells $/ \mathrm{mL}$ and incubated for $72 \mathrm{~h}$. Cells were transferred to microcentrifuge tubes and centrifuged at $600 \mathrm{~g}$ for $3 \mathrm{~min}$. After the supernatant was aspirated, cells were resuspended in $200 \mu \mathrm{L}$ of binding buffer $\left(10 \mathrm{mM}\right.$ HEPES, $150 \mathrm{mM} \mathrm{NaCl}, 1 \mathrm{mM} \mathrm{MgCl}_{2}$, $5 \mathrm{mM} \mathrm{KCl}$, and $\left.1.8 \mathrm{mM} \mathrm{CaCl}_{2}, \mathrm{pH} 7.4\right)$ and transferred to polystyrene test tubes. Two microliters of PI solution were used for each condition, while three microliters of annexin $\mathrm{V}$ were used for each condition. Cells were mixed by vortexing, and data were acquired by using a $\mathrm{BD}$ Fortessa and analyzed by FlowJo.

\section{Cell viability}

Jurkat, Molt-4, and Loucy cells in log growth were seeded at 100,000 cells $/ \mathrm{mL}$ in 96 -well plates in $100 \mu \mathrm{L}$ and incubated for $72 \mathrm{~h}$ in the presence of compounds and fresh media. Ten microliters of CellQuantiBlue reagent was added per well for $2 \mathrm{~h}$ and scanned on a Victor5 Perkin Elmer (Waltham, MA, USA) plate reader (ex550/ em600).

\section{Real-time RT polymerase chain reaction (RT-PCR)}

Molt-4 cells were seeded at 100,000 cells $/ \mathrm{mL}$ in $5 \mathrm{~mL}$. Cells were incubated with appropriate compounds for $72 \mathrm{~h}$. Total RNA was isolated with the TRIZOL (Invitrogen) according to the manufacturer's protocol. The forward primer for NOTCH1 was 5'-AAT GCC TGC CTC ACC AA-3'. The reverse primer for NOTCH1 was 5'-CCA CAC TCG TTG ACA TCC T-3'. The forward primer for $18 \mathrm{~S}$ was: $5^{\prime}$-TAA GTC CCT GCC CTT TGT AAC ACA-3'. The $18 \mathrm{~S}$ reverse primer was 5'-GAT CCG AGG GCC TCA CTA AC-3'. RNA levels were determined with Nanodrop, and cDNA was made by using MMLV reverse transcriptase according to the manufacturer's protocol. SYBR green (Thermo Fisher) was used according to the manufacturer's protocol on a 7500 Applied Biosystems PCR machine. Relative mRNA levels were determined by $2^{-\Delta \Delta \mathrm{Ct}}$ values. 


\section{Luciferase assay}

Cells were electroporated using established settings ${ }^{19}$ with $20 \mu \mathrm{g}$ of pCIneoRL-Notch1 $3^{\prime}$ UTR DNA and $1 \times 10^{7}$ Jurkat cells. Jurkat cells were seeded at $1 \times 10^{5}$ in 24-well plates and treated with compounds. After $72 \mathrm{~h}$, cells were centrifuged at $600 \times g$ for $3 \mathrm{~min}$ and washed with PBS. Cells were lysed with Renilla lysis buffer $(0.5 \times$ PBS, $0.025 \%$ NP-40, 1\% EDTA (w/v), and freshly added $5 \mu \mathrm{M}$ coelenterazine) and sonicated in a water bath, and immediately read on a Victor5 Perkin Elmer (Waltham, MA, USA) plate reader for counts per second. A BCA assay was carried out to determine total protein concentration (Pierce, Waltham, MA, USA).

\section{Western blotting analysis}

Briefly, cells were resuspended in media at 250,000 cells $/ \mathrm{mL}$ for $72 \mathrm{~h}$ with test compounds or solvent controls. Cells were then washed with PBS and lysed in either Whole Cell Lysis buffer (50 mM Tris, pH 8.0, 2\% SDS, and $150 \mathrm{mM} \mathrm{NaCl}$ ) followed by heating at $95^{\circ} \mathrm{C}$ and passage through a $27 \frac{1}{2}$ gauge syringe or RIPA buffer $(25 \mathrm{mM}$ Tris$\mathrm{HCl}, \mathrm{pH} 7.6,150 \mathrm{mM} \mathrm{NaCl}, 1 \% \mathrm{NP}-40,1 \%$ sodium deoxycholate, and $0.1 \%$ SDS) containing freshly added protease and phosphatase inhibitors including aprotinin $(1 \mu \mathrm{g} / \mathrm{mL})$, leupeptin $(1 \mu \mathrm{g} / \mathrm{mL})$, pepstatin $(1 \mu \mathrm{g} / \mathrm{mL})$, PMSF $(200 \mu \mathrm{M})$, sodium vanadate $(200 \mu \mathrm{M})$, sodium diphosphate $(10 \mu \mathrm{M})$, sodium fluoride $(50 \mu \mathrm{M})$, and glycerophosphoric acid $(10 \mu \mathrm{M})$ followed by incubation for $10 \mathrm{~min}$ on ice and centrifugation for $10 \mathrm{~min}$ at $4{ }^{\circ} \mathrm{C}$ at $14,000 \times g$. Lysates were quantified by BCA assay. $1 \times$ SDS was added to the sample, and the sample was then heated for $4 \mathrm{~min}$ at $95^{\circ} \mathrm{C}$ with vortexing. Equivalent masses were loaded onto 7.5 or $12 \%$ bis-acrylamide gels depending on protein size. Proteins were transferred to nitrocellulose membranes, blocked with 5\% BSA in TBS-T, and blotted with primary antibodies in blocking buffer. Alexa-Fluor 680 goat-anti-mouse IgG and IRDye $800 \mathrm{CW}$ goat-antirabbit IgG were used as secondary antibodies in TBS-T.

For the Triton X-114 separations of Rab7, membrane and cytosolic fractions were purified as described previously with some modifications ${ }^{19}$. Briefly, cells were resuspended in media at 250,000 cells $/ \mathrm{mL}$ and treated with DGBP and/or test compounds for $72 \mathrm{~h}$. After PBS wash, cells were lysed in Triton X-114 lysis buffer and processed for Western blots. Gels were loaded based on equivalent cell numbers/volumes.

\section{Statistical analysis}

One-way or two-way ANOVA was used to determine statistical significance as indicated in the figure legends. In either case, follow-up tests to compare individual treatments were done by using the Tukey method in GraphPad Prism. Control conditions were compared with treatment conditions or between pairs of conditions indicated in the graphs. An $\alpha$ of 0.05 was used to establish significance. Bar and line graphs represent mean \pm SD. Experiments were replicated at least 3 times as indicated $(n=3)$.

\section{Results \\ DGBP and lovastatin, but not zoledronate or DAPT, inhibit T-ALL proliferation}

A recent report demonstrated that Rab GTPases regulate Notch1 processing ${ }^{11}$. Because Rab proteins are posttranslationally geranylgeranylated, we asked whether geranylgeranylation inhibitors would affect Notchdependent cells. We treated the T-ALL cell lines Molt-4 and Jurkat with the isoprenoid biosynthesis inhibitors lovastatin (inhibitor of HMG-CoA reductase), zoledronate (inhibitor of farnesyl diphosphate synthase), and digeranyl bisphosphonate (DGBP) (inhibitor of geranylgeranyl diphosphate synthase) ${ }^{13}$ (Fig. 1a) to investigate the effect on cell viability. Because zoledronate inhibits bone resorption and is active in cells of bone origin, as a control, we first assessed the impact of zoledronate and digeranyl bisphosphonate on U2OS osteosarcoma cells. Here, zoledronate was more potent than DGBP with strong activity at $10 \mu \mathrm{M}$, while DGBP only partially inhibited proliferation at $100 \mu \mathrm{M}$ (Fig. 1b). In contrast, in the T-ALL cells, both DGBP and lovastatin decreased cell viability with DGBP demonstrating the stronger effect (Fig. 1c, d). Zoledronate did not impact Molt-4 and Jurkat cell viability at concentrations up to $100 \mu \mathrm{M}$. Thus, the pattern of zoledronate and DGBP activity is reversed in T-ALL cells in contrast to osteosarcoma. Although the active cellular concentrations are in the micromolar range, these concentrations are expected to be clinically achievable in the bone marrow environment due to the distribution kinetics driven by the bisphosphonate substructure. These values are also typical of charged bisphosphonates that require uptake by endocytic mechanisms ${ }^{32}$ and transporters ${ }^{33}$. We also evaluated the $\gamma$-secretase inhibitor DAPT, which has been proposed as a route to disrupting Notch1 processing and function. Consistent with the literature ${ }^{34,35}$, DAPT did not strongly impact cell viability in this time frame (Fig. 1c, d).

\section{GGDPS inhibition decreases expression of the Notch1 NICD and alters Rab7 localization}

Because Notch1 is a critical oncogene that drives proliferation of T-ALL cells and it is required for proliferation of T-ALL cell lines including Jurkat cells ${ }^{36,37}$, we evaluated these compounds in Western blots to determine the effect on Notch1 expression. Of this panel, only DGBP completely and dose-dependently decreased Notch1 protein expression and levels of the Notch1 NICD in both Jurkat (Fig. 2a) and Molt-4 cells (Fig. 2b). The bands visualized are the predominant band on Notch1 Western 

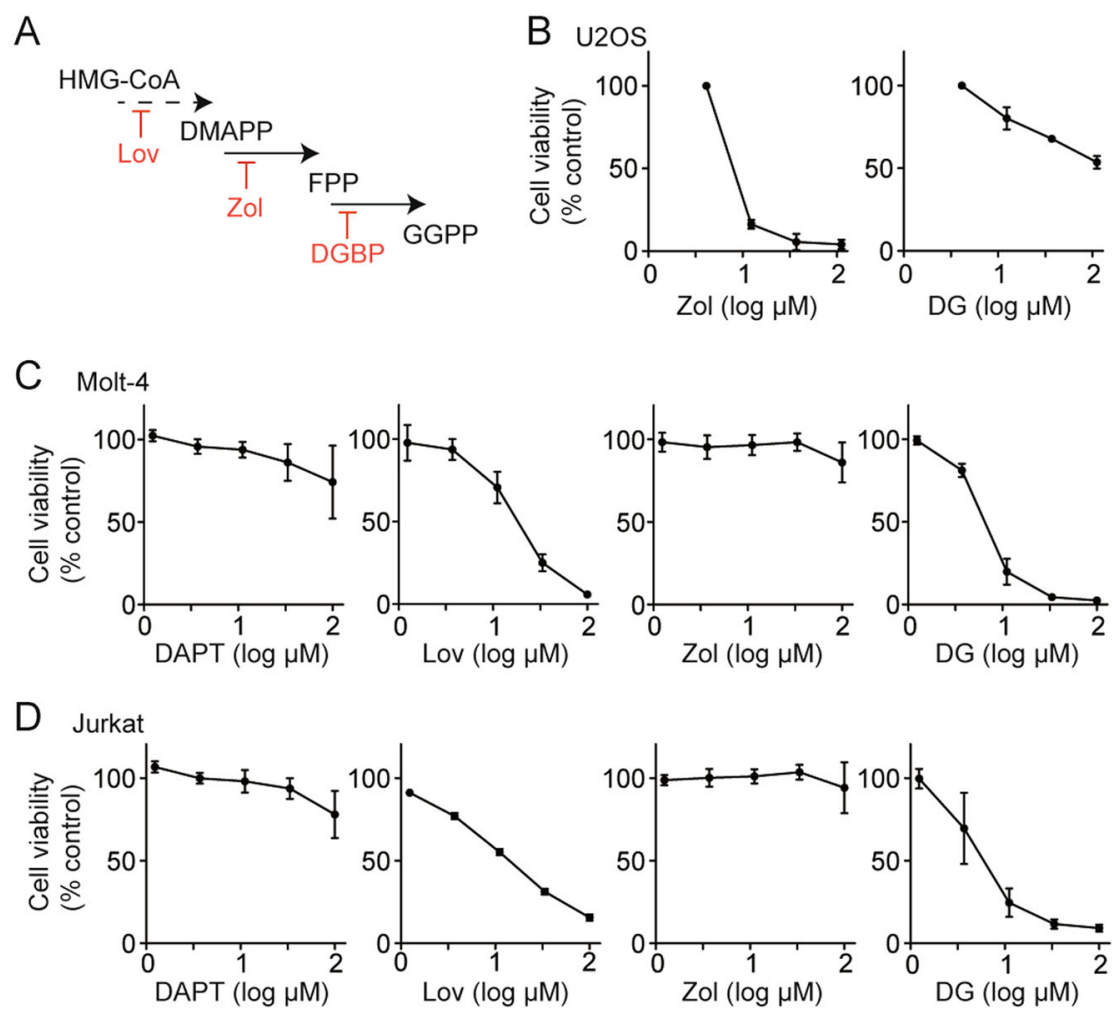

Fig. 1 Impact of mevalonate pathway inhibitors and a $\mathbf{y}$-secretase inhibitor on cell viability. a Mevalonate pathway inhibitors used in this study. HMG-CoA reductase is inhibited by lovastatin (Lov), farnesyl diphosphate synthase is inhibited by zoledronate (Zol), and geranylgeranyl diphosphate synthase is inhibited by DGBP. $\mathbf{b}$ Impact of prenyl synthase inhibitors on viability of U2OS osteosarcoma cells. Graphs display means and standard deviations of two independent experiments for each compound. c, d Cell viability of Molt-4 (c) and Jurkat (d) cells after $72 \mathrm{~h}$ of treatment with the $\gamma$-secretase inhibitor DAPT or the mevalonate pathway inhibitors lovastatin, zoledronate, or DGBP. Graphs display means and standard deviations of three independent experiments for each compound and cell line

blots by using the bTAN20 antibody $(\sim 88 \mathrm{kDa})$, corresponding to the predicted size of the human NICD $(86 \mathrm{kDa})^{38}$. Both cell lines were resistant to $\gamma$-secretase inhibition (Fig. 1), and indeed no inhibitory effect was observed on the Notch1 NICD at concentrations up to $100 \mu \mathrm{M}$ of DAPT in either cell line. However, use of a second Notch1 antibody specific to a cleaved form of Notch1 revealed an additional band at $110 \mathrm{kDa}$ in Jurkat cells that was impacted by DAPT treatment. The $110-\mathrm{kDa}$ band was not observed in the Molt- 4 cells, and it was also not observed through probing with the bTAN20 antibody. Taken together, DAPT treatment increases the $88-\mathrm{kDa}$ form of Notch1 and decreases the 110-kDa form of Notch1 with no impact on viability, while treatment with DGBP decreases both forms of Notch1 and impacts cell viability.

As predicted, DGBP also increased the amount of the unprocessed non-geranylgeranylated Rab7 GTPase as indicated by a slight upward shift in the apparent molecular weight (Fig. 2a, b). Because the band shift is subtle and difficult to observe/interpret, we additionally performed cellular fractionation experiments to ensure that
Rab7 localization was impacted by DGBP as desired (Fig. 2c). Here, DGBP dose-dependently decreased the fraction of Rab7 associated with the membrane while increasing the fraction of Rab7 in the cytoplasm. Thus, DGBP both depletes Notch1 NICD and impacts Rab7 processing and localization. The impact of DGBP on proliferation, Notch1 NICD expression, and Rab7 processing occurs within the $10-30 \mu \mathrm{M}$ range.

\section{GGDPS inhibition decreases expression of Notch1 in other hematological cell lines}

In order to assess the prevalence of Notch1 regulation by GGDPS, we examined the impact of DGBP treatment on other non-T-ALL cell lines. Treatment with DGBP in various hematological cell lines revealed that DGBP reduces Notch1 NICD in cell lines expressing Notch1 (Fig. 2d). In Loucy cells, there is low Notch1 expression and no effect of DGBP on levels of NICD. However, RPMI-8226, K562, and to a lesser extent HL-60 cells expressed NICD that decreased with treatment of DGBP. Daudi cells had no expression of Notch1. Overall, in some non-T-ALL hematologic cell lines that express NICD, 


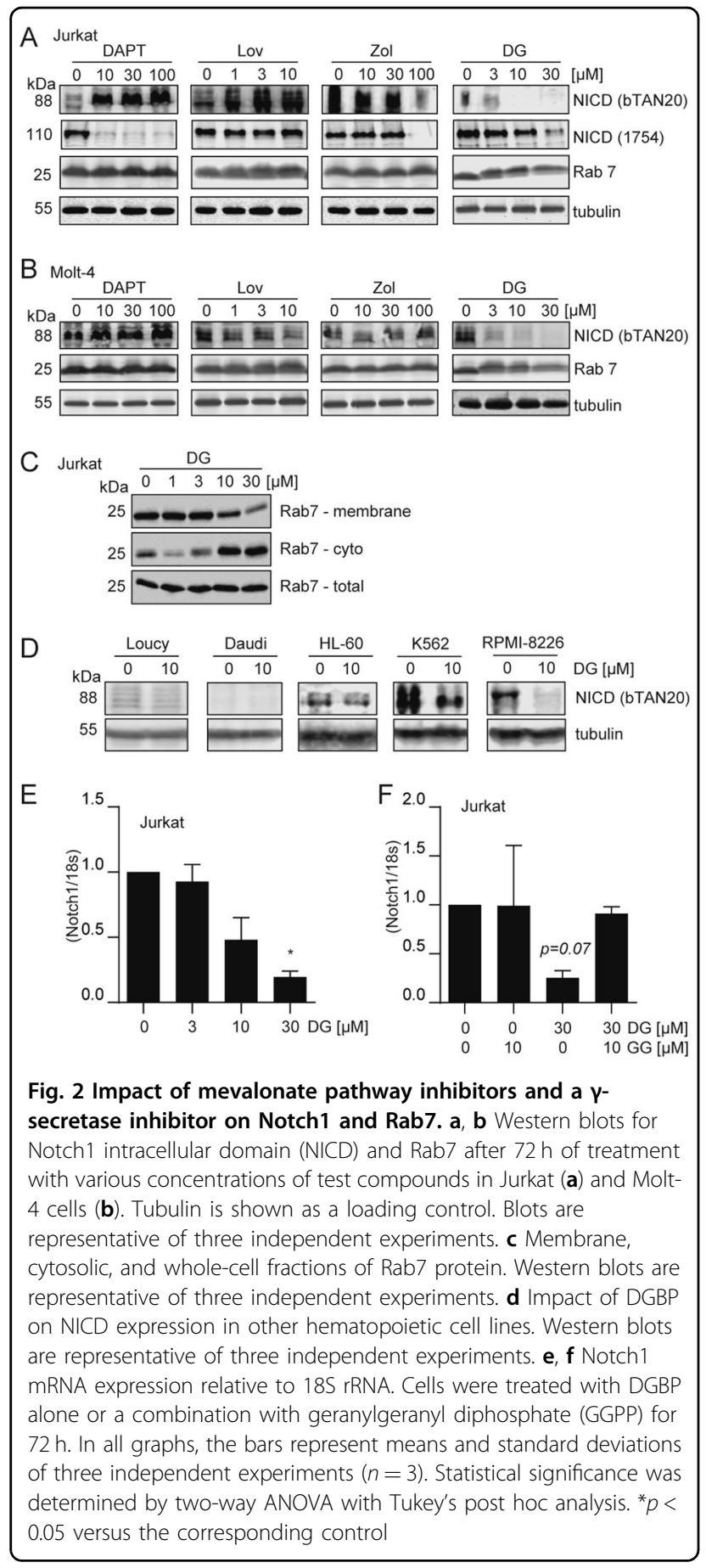

treatment with DGBP reduces Notch1 expression indicating that this is a conserved phenomenon, though it is most important in T-ALL cells given their dependence on Notch1.

\section{GGDPS controls Notch1 mRNA expression}

We were surprised that inhibition of GGDPS would have such a strong effect on Notch1 expression, and asked
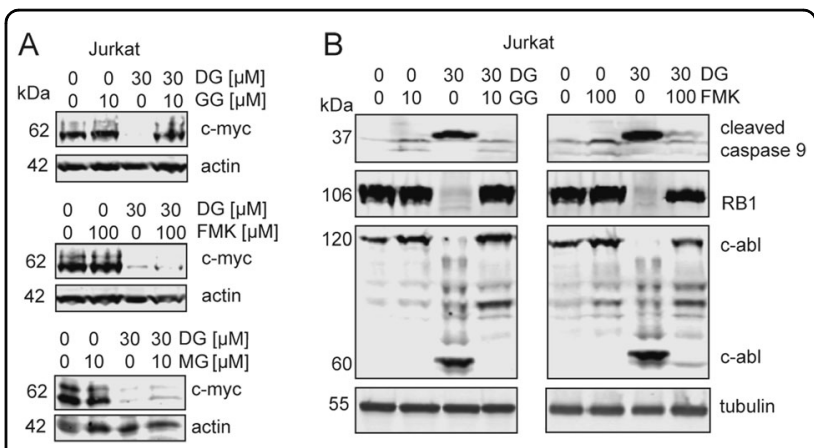

Fig. 3 DGBP impacts c-myc expression independent of caspases and impacts RB and c-abl expression dependent upon caspases. a Western blots showing c-myc with DGBP treatment and cotreatment of GGPP, Z-VAD-FMK, or MG132 in Jurkat cells. Actin is shown as a loading control. All Western blots are representative of three independent experiments. $\mathbf{b}$ Western blot analysis of cleaved caspase 9, retinoblastoma (RB1), and c-abl. Tubulin is shown as a loading control. Jurkat cells were treated for $72 \mathrm{~h}$ with DGBP in the presence or absence of GGPP or Z-VAD-FMK. Western blots are representative of three independent experiments

if additional gene expression mechanisms were involved. A prior study had shown that GGDPS inhibition can also impact mRNA expression through increased flux of the reactant FPP into sterol synthesis ${ }^{39}$. To examine whether GGDPS inhibition could also impact Notch1 expression at the mRNA level, we tested whether or not DGBP could impact the mRNA expression of Notch1 by using realtime PCR (Fig. 2e, f). Here, treatment with DGBP dosedependently reduced Notch1 total mRNA expression (Fig. 2e). The effect of DGBP was prevented by coincubation with GGPP (Fig. 2f). Therefore, DGBP reduces Notch1 expression at the mRNA level through specific engagement of GGDPS. Both Notch1 mRNA reduction and alteration of Rab7 localization happen at similar concentrations and may contribute to the impact of DGBP on Notch1.

\section{GGDPS inhibition decreases c-myc expression}

We next sought to understand how reduced Notch1 expression caused by GGDPS inhibition would impact factors downstream of Notch1. Notch1 affects transcription of a variety of genes, notably including c-myc $(M Y C)$, which promotes oncogenesis in T-cell leukemia ${ }^{10,40}$. We treated Jurkat cells with DGBP and co-treated with GGPP or the caspase inhibitor Z-VAD-FMK. GGDPS inhibition decreased levels of c-myc protein expression, and cotreatment with GGPP restored the levels of c-myc (Fig. 3a). Co-treatment with Z-VAD-FMK did not rescue the effect of DGBP on c-myc (Fig. 3a), indicating that the impact on the Notch/myc axis is not downstream of caspase activation. Co-treatment of DGBP with the proteasome inhibitor, MG132, did not rescue the effect of DGBP on c-myc depletion, consistent with transcriptional 
regulation of c-myc downstream of Notch1 rather than increased c-myc protein degradation (Fig. 3a). Therefore, GGDPS inhibition reduces expression of Notch1 and transcription of the Notch1 effector c-myc in a way that is upstream or independent of cleaved caspases.

\section{GGDPS inhibition impacts signaling to caspases and apoptosis}

To determine how GGDPS inhibition affects caspases, we treated Jurkat cells with DGBP and either GGPP or ZVAD-FMK (Fig. 3b). As expected, DGBP treatment increased the expression of cleaved caspase 9. The effect of DGBP on cleaved caspase 9 was fully rescued by GGPP and Z-VAD-FMK. The pattern was also observed with retinoblastoma protein (RB1) expression. Furthermore, DGBP treatment decreased the $120-\mathrm{kDa}$ fraction of $\mathrm{c}-\mathrm{abl}$ and increased expression of the $60-\mathrm{kDa}$ cleavage product of c-abl. The effect on c-abl was rescued by co-treatment with GGPP and by co-treatment with Z-VAD-FMK. Therefore, the effects of DGBP on caspase 9, RB1, and cabl were all rescued by Z-VAD-FMK and are downstream of caspase activation. While c-abl activation is thought to be upstream of cleaved caspases in some circumstances, cleaved caspases have been previously shown at times to cleave $\mathrm{c}-\mathrm{abl}{ }^{41}$. Thus, DGBP allows for Jurkat cells to enter apoptosis through activation of caspases, depletion of retinoblastoma, and stimulation of caspase-mediated cabl cleavage.

\section{c-abl mediates apoptosis induced by GGDPS inhibition downstream of caspases in T-ALL}

Surprised that c-abl was acting downstream of caspases in this system, we wanted to further assess the role of cabl in apoptosis induced by GGDPS inhibition. We cotreated cells with DGBP and the c-abl inhibitors imatinib or nilotinib and determined their impact on viability, apoptosis, and protein expression. Imatinib dosedependently reduced the impact of DGBP on Molt-4 viability with a peak impact of around $10 \mu \mathrm{M}$ and $\mathrm{EC}_{50}$ of $3.5 \mu \mathrm{M}$ (Fig. 4a). Both imatinib and nilotinib reduced the severity of DGBP on proliferation in Jurkat and Molt-4 cells but not in Loucy cells (Fig. 4b), which do not express Notch1 (Fig. 2c). We assessed the degree of apoptosis by Annexin V staining. Here, DGBP-induced apoptosis was blocked by imatinib in both Jurkat and Molt-4 cells (Fig. 4c). Co-treatment with imatinib also restored expression of the $120-\mathrm{kDa}$ form of $\mathrm{c}-\mathrm{abl}$ and partially reduced expression of the $60-\mathrm{kDa}$ form (Fig. 4d). The effect of DGBP on cleaved caspase 9 was also partially rescued by imatinib co-treatment. Co-treatment with imatinib did not affect the ability of GGDPS inhibition to reduce expression of the $88-\mathrm{kDa}$ Notch1 band (Fig. 4e). Together, c-abl acts downstream or independently of Notch1 to mediate DGBP-induced apoptosis; however, it

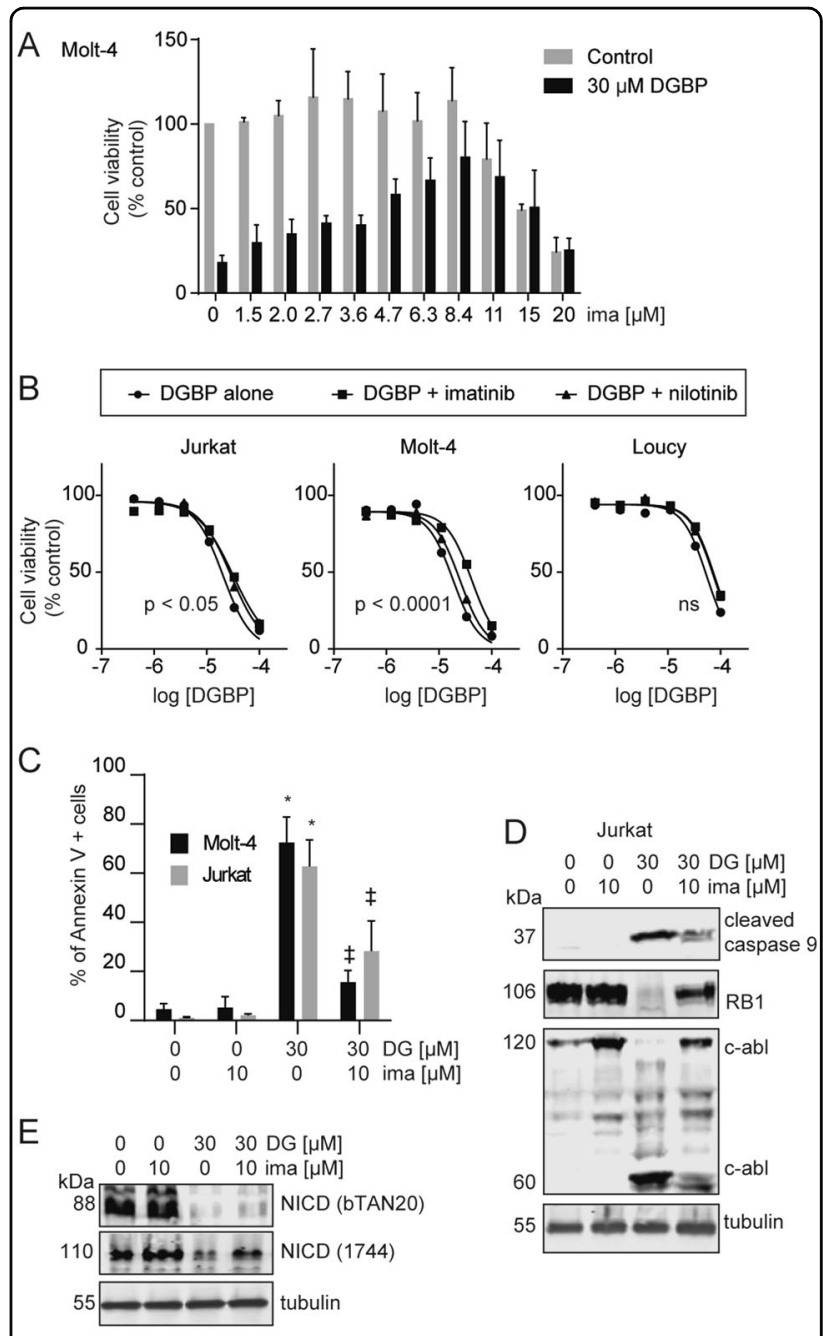

Fig. 4 Inhibitors of c-abl are protective against apoptosis induced by DGBP, downstream of Notch1 and caspases. a Dose response of Molt-4 cell viability after treatment with or without $30 \mu \mathrm{M}$ DGBP and the indicated concentrations of imatinib for $72 \mathrm{~h}$. $\mathbf{b}$ Dose response of Molt-4, Jurkat, and Loucy cell viability with DGBP in combination with either $10 \mu \mathrm{M}$ imatinib or $10 \mu \mathrm{M}$ nilotinib for $72 \mathrm{~h}$. The $X$ axis represents the log of the molar concentration. Curves were generated by nonlinear regression by using a variable slope fourparameter model. The indicated $p$ values represent confidence in whether the $I C_{50}$ values differed between conditions. c Molt- 4 or Jurkat cells were treated with DGBP with or without imatinib for $72 \mathrm{~h}$ and assessed by Annexin $V$ staining. Flow plots shown are representative of three independent experiments. The bars represent means and standard deviations of three independent experiments $(n=3)$. The results were analyzed by using two-way ANOVA with Tukey's post hoc analysis. ${ }^{*} p<0.05$ versus untreated controls. ${ }^{*} p<0.05$ versus DGBP-treated condition. $\mathbf{d}$ Western blotting analysis of cleaved caspase 9, retinoblastoma, and c-abl. Tubulin is shown as a loading control. Jurkat cells were treated for $72 \mathrm{~h}$ with DGBP in the presence or absence of imatinib. Western blots are representative of three independent experiments. e Western blot analysis of NICD. Tubulin is shown as a loading control. Jurkat cells were treated for $72 \mathrm{~h}$ with DGBP in the presence or absence of imatinib. Western blots are representative of three independent experiments 
may have functions both upstream and downstream of caspases.

\section{GGDPS inhibition increases the DNA-damage response}

A recent report demonstrated that Notch1 can directly negatively regulate the DNA-damage response ${ }^{42}$. The DNA-damage response in Jurkat cells treated with DGBP is higher than that of primary $\mathrm{T}$ cells treated with DGBP as demonstrated by phosphorylated histone H2A.X (Fig. 5a). The effect of DGBP on p-H2A.X was rescued by GGPP, but not by Z-VAD-FMK or imatinib (Fig. 5b). Because a study by Vermezovic et al. found that Notch1 binds to the kinase regulatory domain of ataxia telangiectasia mutated (ATM), and prevents ATM activation ${ }^{42}$, we set out to determine if GGPP depletion regulates Notch1 to induce ATM activation and the DNA-damage response. By using an ATM inhibitor (Ku55933), we tested whether ATM was required for the apoptotic effects of GGDPS inhibition. DGBP treatment dose-dependently decreased ATM expression (Fig. 5c). Treatment of Molt-4 and Jurkat cells with Ku55933 in combination with DGBP reduced the apoptosis induced by DGBP (Fig. 5d). Ku55933 also dose-dependently reduced the impact of DGBP on Molt-4 viability with a peak impact of around $12 \mu \mathrm{M}$ and $\mathrm{EC}_{50}$ of $6.8 \mu \mathrm{M}$ (Fig. 5e). At concentrations below the $\mathrm{EC}_{50}$, the combination of imatinib and Ku55933 was additive (Fig. 5f).

\section{Notch1 depletion and cellular apoptosis induced by GGDPS inhibition are restored by $\mathrm{Y}$-secretase inhibition}

To further assess the relationship between GGDPS and Notch1 in T-ALL cells, we hypothesized that cotreatment with DGBP and DAPT would more strongly impact proliferation and viability than either agent alone. On the contrary, co-incubation with DGBP and DAPT actually improved cell viability relative to treatment with DGBP alone. In this regard, both DAPT and a second $\gamma$ secretase inhibitor MK-0752 dose-dependently reduced the effect of DGBP on apoptosis (Fig. 6a, b). Thus, in these T-ALL cell lines, $\gamma$-secretase works against viability and secretase inhibitors improve viability.

We next examined how this combination would affect expression of Notch1. First, as controls, we found that as expected, GGPP antagonized the reduction in Notch1 expression caused by DGBP (Fig. 6c), while co-incubation with Z-VAD-FMK did not impact the ability of DGBP to decrease NICD expression (Fig. 6d). This is further evidence that the impact of GGDPS inhibition on Notch1 expression is not a generalized nonspecific phenomenon downstream of caspase activation. To determine whether or not GGDPS reduces Notch1 expression upstream or downstream of $\gamma$-secretase cleavage, we co-treated cells with the $\gamma$-secretase inhibitor (DAPT) in combination with DGBP. DAPT restored protein levels of the $88-\mathrm{kDa}$ band of Notch1 with DGBP co-incubation in both Molt-4 (Fig. 6e) and Jurkat cells (Fig. 6f). The 110-kDa Notch1 band was decreased in Jurkat cells by both DAPT and DGBP (Fig. 6f). Therefore, DGBP reduces expression of both the 88- and the 110-kDa Notch1 band and decreases cell viability, while DAPT changes the relative amounts of the 88- and the 110-kDa Notch1 band and improves cell viability.

\section{GGDPS controls Notch1 mRNA expression, while secretase and ATM, but not GGDPS or c-abl, control Notch1 mRNA degradation}

Because GGDPS inhibition had affected Notch1 expression through impacts on both its processing and its mRNA expression (Fig. 2), we investigated whether $\gamma$ secretase inhibition would also impact Notch1 mRNA expression. In this regard, treatment with DAPT alone had no impact on Notch1 total mRNA expression (Fig. 7a). Surprisingly, the effect of DGBP on Notch1 mRNA expression was prevented by co-incubation with DAPT (Fig. 7a). This indicates a role for $\gamma$-secretase in regulating not only the processing of Notch 1 but also its gene expression.

Notch 1 mRNA expression is regulated by miR-200 interaction with the Notch1 $3^{\prime}$ UTR, which can be analyzed with a luciferase reporter construct that contains the luciferase gene flanked by the Notch $13^{\prime} \mathrm{UTR}^{31}$. We assessed the ability of DGBP and the rescue agents to affect the miR-200-mediated regulation of Notch1 mRNA degradation. Treatment with DGBP did not significantly alter Notch1 mRNA stability in this system (Fig. 7b), meaning that the DGBP-induced reduction of Notch1 mRNA expression is not due to increased degradation. Treatment with imatinib also did not impact Notch1 mRNA degradation. However, treatment with DAPT and Ku55933 decreased degradation of luciferase mRNA fused to the Notch1 $3^{\prime}$ UTR leading to elevated luciferase activity. These data suggest that miR-200 not only regulates Notch, but also that miR-200 is itself regulated by secretase and ATM.

\section{Discussion}

Notch proteins are critical to development and progression of cancers including T-ALL. In this study, we demonstrate that inhibition of the enzyme GGDPS reduces Notch1 expression and impacts factors known to be downstream of Notch1. These experiments provide evidence for regulation of Notch1 by lipid synthesis, and disruption of this mechanism can impact Notch1 expression and viability of Notch1-dependent cells.

DGBP is more effective than the secretase inhibitor DAPT and the other isoprenoid biosynthesis inhibitors at decreasing cell viability (Fig. 1) and levels of NICD (Fig. 2). The decrease in NICD is likely a result of at least two 
A

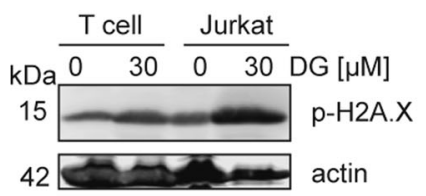

B
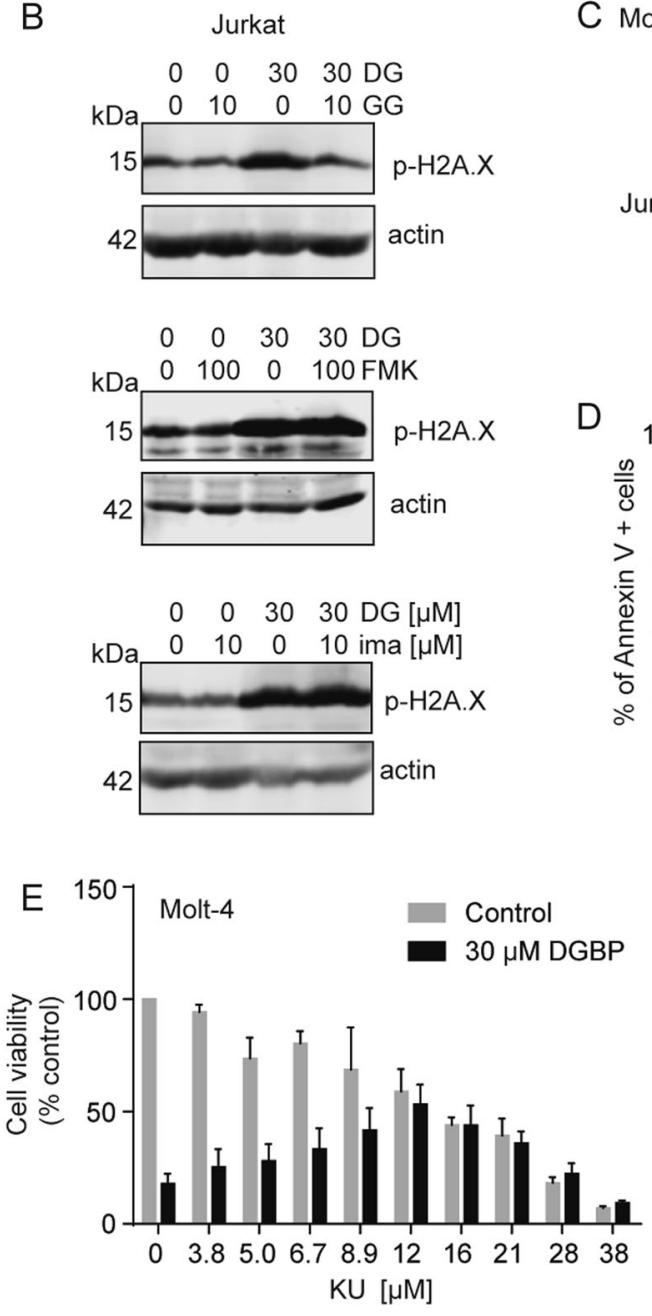

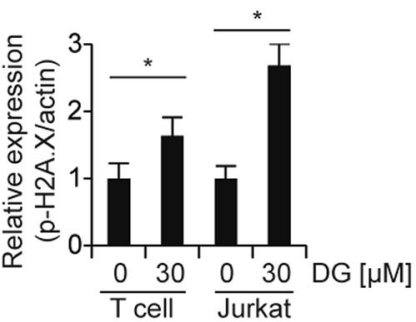

C Molt-4

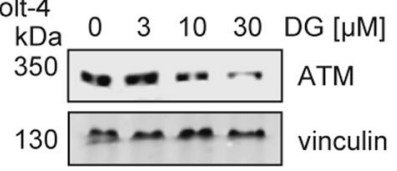

Jurkat

$\mathrm{kDa} \quad 0 \quad 3 \quad 10 \quad 30 \quad \mathrm{DG}[\mu \mathrm{M}]$

$350-2-\ldots$ ATM

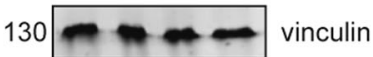

D
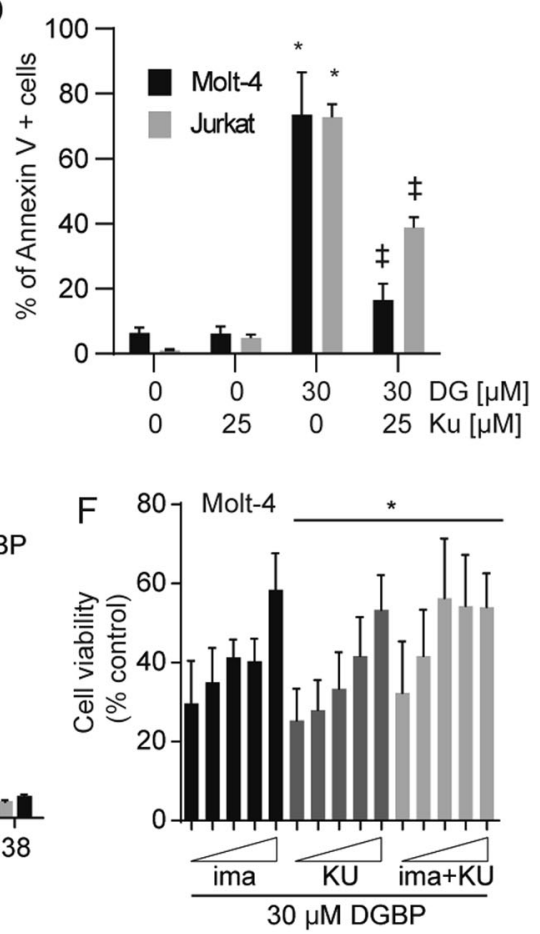

Fig. 5 DGBP-induced apoptosis is mediated by ATM. a Western blot analysis of phosphorylated H2A.X in either expanded primary T cells or Jurkat cells after $72 \mathrm{~h}$ of treatment with DGBP, with actin shown as a loading control. $\mathbf{b}$ Western blotting analysis of p-H2A.X. Actin is shown as a loading control. Jurkat cells were treated for $72 \mathrm{~h}$ with DGBP in the presence or absence of GGPP, Z-VAD-FMK, or imatinib. Western blots are representative of three independent experiments. c Western blot analysis of ATM expression in Molt-4 or Jurkat cells treated with DGBP for $72 \mathrm{~h}$, with vinculin as a loading control. d Annexin V staining showing $72 \mathrm{~h}$ of DGBP treatment along with co-treatment with or without the ATM inhibitor Ku55933 in Molt-4 and Jurkat cells. The bars represent means and standard deviations of three independent experiments $(n=3)$. e Dose response of Molt- 4 cell viability after treatment with or without $30 \mu \mathrm{M}$ DGBP and the indicated concentrations of Ku55933 for $72 \mathrm{~h}$. The bars represent means and standard deviations of three independent experiments $(n=3)$. $\mathbf{f}$ Dose response of imatinib, Ku55933, or the combination of both inhibitors. The bars represent means and standard deviations of three independent experiments $(n=3)$. Panel $(\mathbf{a})$ was analyzed by using a T test, while panels $(\mathbf{d}, \mathbf{e})$ were analyzed by using two-way ANOVA with Tukey's post hoc analysis. ${ }^{*} p<0.05$ versus controls. ${ }^{\ddagger} p<0.05$ versus DGBP-treated condition 

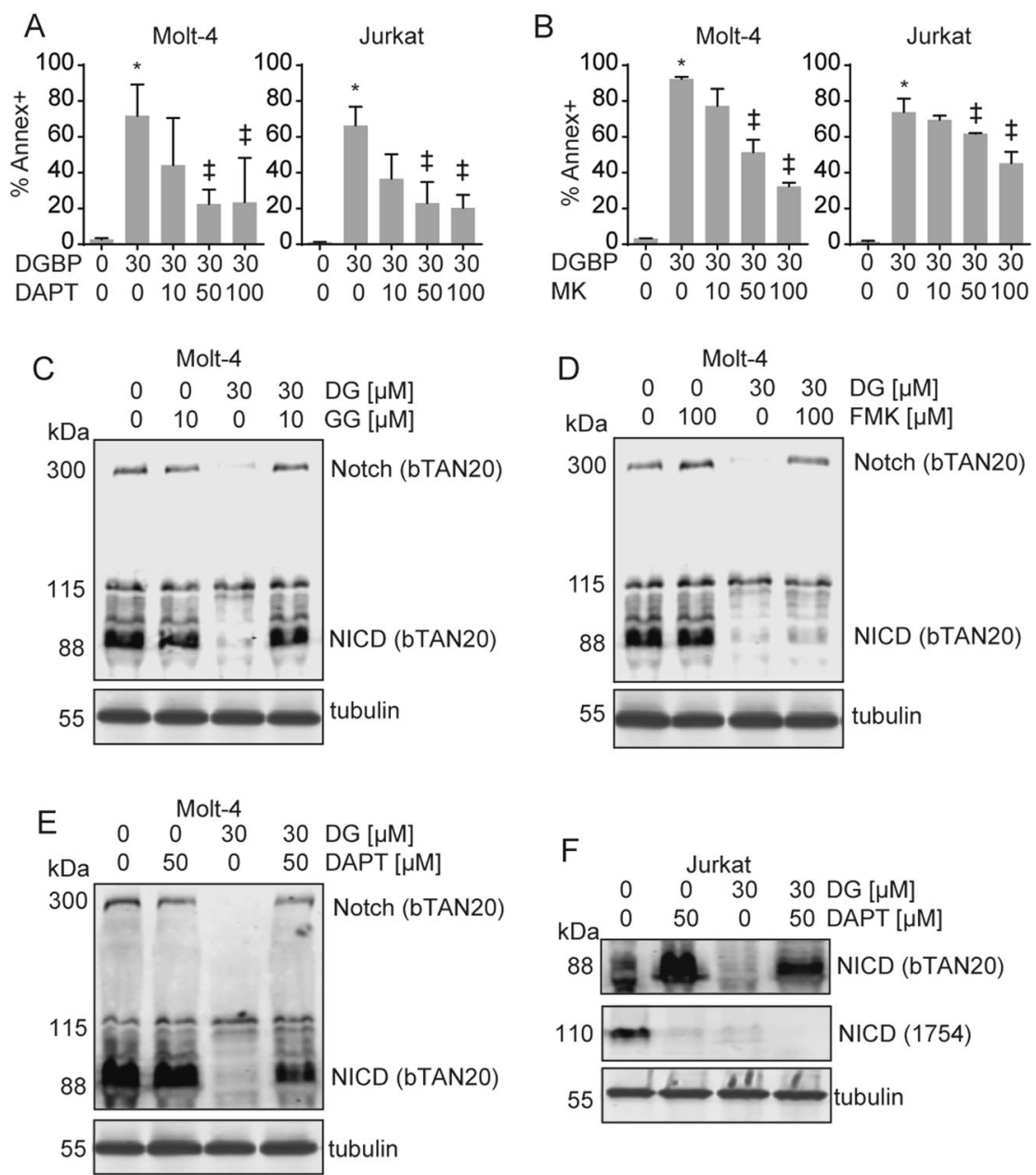

Fig. 6 Secretase inhibitors rescue the effect of DGBP on apoptosis. $\mathbf{a}$, b Annexin V staining of Molt-4 or Jurkat cells treated with DGBP and cotreated with or without (a) DAPT or (b) MK-0752 for $72 \mathrm{~h}$. Graphs represent means and standard deviations of three independent experiments. Statistical significance was determined by two-way ANOVA with Tukey's post hoc analysis. ${ }^{*} p<0.05$ versus untreated controls. ${ }^{\ddagger} p<0.05$ versus DGBPtreated condition. $\mathbf{c}, \mathbf{d}$ Western blot of full-length Notch 1 and NICD in Molt-4 cells following $72 \mathrm{~h}$ of treatment with DGBP and co-treatment with or without either the GGDPS product GGPP (c) or the caspase inhibitor Z-VAD-FMK (d). Tubulin is shown as a loading control. e, $\mathbf{f}$ Western blots of Notch 1 and NICD after $72 \mathrm{~h}$ of treatment with DGBP and co-treatment with or without DAPT in Molt-4 (e) and Jurkat (f) cells. All Western blots are representative of three independent experiments

underlying mechanisms-(1) inhibition of posttranslational processing of Rab proteins that are required for Notch1 trafficking, and (2) altered transcription of Notch1 itself. Immediately downstream effects include reduced c-myc expression, increased pH2A.X, and decreased ATM expression, resulting in caspase-dependent apoptosis that is associated with decreased retinoblastoma expression and cleaved c-abl (Fig. 3). Using GGDPS inhibitors to target Notch1 signaling may be a promising therapeutic strategy because Rab GTPases are required for Notch1 processing $^{11}$. While direct inhibitors of $\gamma$-secretase may prevent Notch1 signaling in some cases, certain mutations to Notch1 may confer insensitivity to secretase processing.
Because Rab GTPases are expected to be involved in both normal and mutant Notch1 processing, disrupting GTPase function via inhibition of GGDPS allows for Notch1 disruption even in the event of mutations to Notch1.

Because $\gamma$-secretase has been proposed as an antiNotch1 target, we evaluated combinations of secretase inhibitors and DGBP to look for potential synergies. We surprisingly found that DAPT reduces the ability of DGBP to promote apoptosis (Fig. 6), and Notch1 mRNA degradation is regulated by DAPT. The impact of DGBP and DAPT on NICD expression is more nuanced. In agreement with a prior study ${ }^{43}$, treatment with DAPT not only decreases expression of the NICD detected by the 


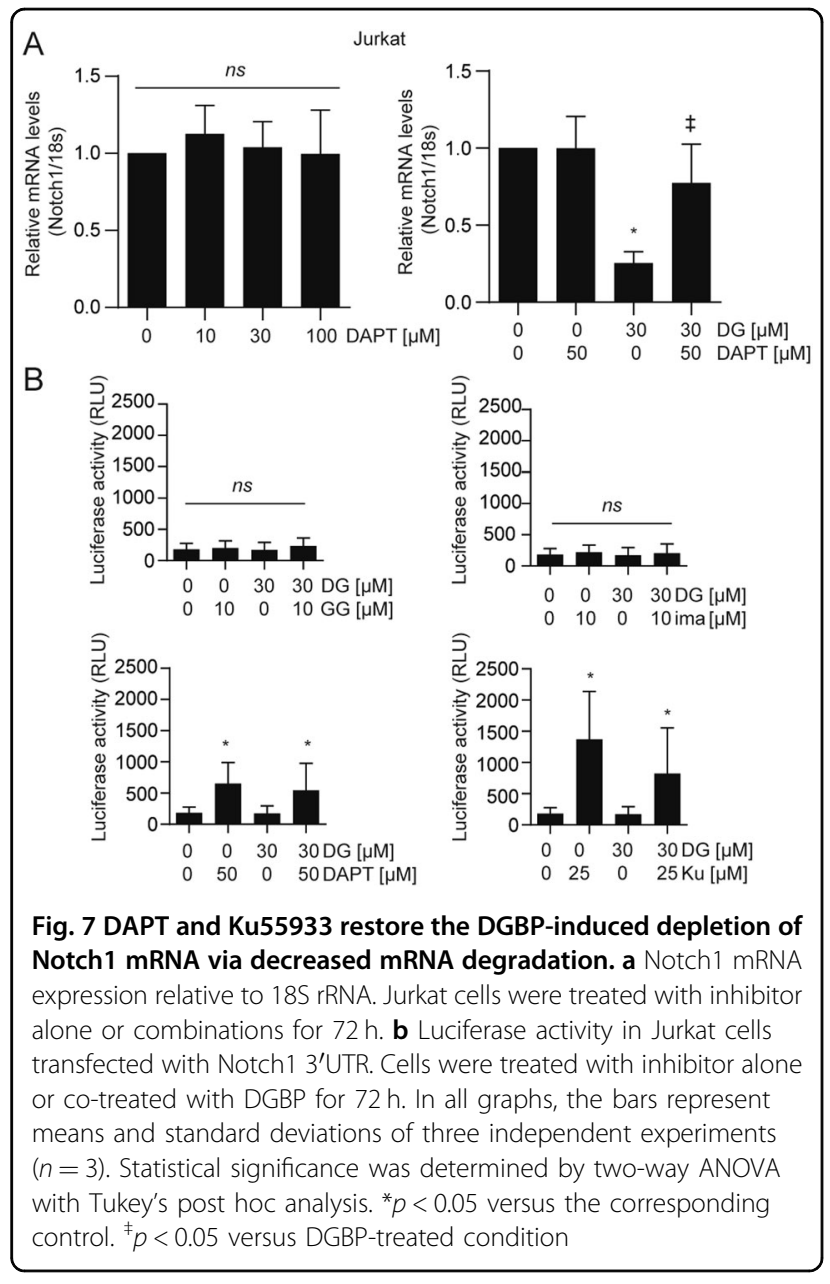

cleaved Notch1 antibody as expected, but also increases expression of NICD detected by a total Notch1 antibody. DGBP clearly reduces the NICD detected by both antibodies. In combination, the effect of DAPT on NICD expression can overcome the effect of DGBP, which correlates with the ability of DAPT to reduce DGBPinduced apoptosis. Therefore, GGDPS inhibition depletes NICD, while a $\gamma$-secretase inhibitor, DAPT, alters NICD expression. We speculate that this may occur because DAPT is a non-transition-state inhibitor of the $\gamma$-secretase complex that does not bind to the active site of the $\gamma$ secretase complex protease presenilin-1, but rather to an allosteric site within presenilin- $1^{8}$. Because there are mutations along the transmembrane region of Notch $1^{44}$, the binding conformation of the mutated $\alpha$-helix may be different from the wild type. Binding of DAPT in these cases may allow for allosteric modulation rather than inhibition of the catalytic domain. Interestingly, there is overlap between the reported binding site of DAPT on presenilin-1 (aa372-467) ${ }^{45}$ and the binding site of Rab11 on presenilin-1 (aa374-400) ${ }^{46}$. Perhaps, binding of DAPT mimics binding of Rab to enable secretase processing of Notch1-though further experiments would be needed to confirm this hypothesis.

There is evidence in favor of repurposing isoprenoid biosynthesis inhibitors such as lovastatin and zoledronate for anticancer applications because zoledronate is currently used in the clinic for metastatic bone disease, and these inhibitors can lead to apoptosis ${ }^{47,48}$. Originally developed for non-cancer applications, these drugs have generated much interest for their potential anticancer properties. For example, a recent article showed that statins could sensitize leukemia cells to venetoclax, a BCL-2 inhibitor, in a way that is dependent upon GGPP depletion ${ }^{49}$. Interestingly, we observed GGDPS inhibition to affect Notch1 more strongly than HMG-CoA reductase and farnesyl diphosphate synthase inhibition ${ }^{18,50}$. One potential explanation for this phenomenon is that, unlike GGDPS, both HMG-CoA reductase and farnesyl diphosphate synthase are under transcriptional control through sterols $^{51}$, which can mitigate the effects of these agents $^{39,52,53}$. Alternatively, they may differ in rates of uptake depending on the cell line. As zoledronate has a higher charge:mass ratio than DGBP, its cellular entry is more likely to rely on endocytosis ${ }^{32}$.

We show that once DGBP reduces Notch1 expression, there is a twofold effect on downstream signaling: (1) decreased transcription of c-myc, and (2) induction of p$\mathrm{H} 2 \mathrm{AX}$, a marker of the DNA-damage response. C-myc is a well-known downstream target of Notch1 in TALL ${ }^{10,40,54}$, while Notch1 has been reportedly involved in the DNA-damage response ${ }^{42}$. A potential mechanism is that DGBP-induced depletion of the NICD may free ATM to become activated and phosphorylate H2A.X, a marker for DNA damage ${ }^{55}$. While our studies did show rescue of DGBP-induced apoptosis by an ATM inhibitor, it remains possible that the inhibitor could disrupt function of other kinases affecting H2A.X, and further studies would be needed to conclusively determine whether ATM is mediating phosphorylation of H2A.X in this system. We propose that GGPP depletion does not directly increase DNA damage, but rather allows for cells to recognize the level of DNA damage. In this scenario, ATM would then activate cleaved caspases and initiate apoptosis ${ }^{56}$, while caspases would cleave retinoblastoma protein, which sits on the catalytic domain of c-abl and prevents its activation $^{57}$. There is some precedence for Notch1 involvement in retinoblastoma protein degradation as a $\gamma$-secretase inhibitor regulates retinoblastoma to exit the cell cycle ${ }^{34}$. Furthermore, lovastatin and nitrogenous bisphosphonates regulate retinoblastoma protein via hypophosphorylation ${ }^{58}$. This reduction in retinoblastoma levels allows for caspases to cleave c-abl ${ }^{41,59}$. Alternatively, c-abl has been previously shown to be part of an ATM complex that activates in response to ionizing radiation ${ }^{60}$. 
However, in our studies, imatinib was not able to rescue the effect of DGBP on p-H2A.X, while the caspase inhibitor did rescue the effect of DGBP on c-abl cleavage, suggesting that the major effect of abl is downstream of caspases in this system. Together, these data indicate the connection between disruption of geranylgeranylation and induction of apoptosis in T-ALL.

Finally, we found that GGPP depletion decreases Notch1 mRNA levels but does not affect Notch1 mRNA degradation. miR-200 interacts with Notch1 signaling in cancer stem cells in breast cancer ${ }^{61}$ and pancreatic cancer $^{62}$. Interestingly, only DAPT and Ku55933 impact Notch1 mRNA degradation. We predict that both of these inhibitors act on miR-200 through ATM inhibition with Ku55933 directly inhibiting ATM and DAPT acting indirectly. Further studies aimed at understanding the impact of secretase and ATM inhibitors on Notch1 expression in the context of anti-proliferative agents such as DGBP would certainly be of interest.

\section{Acknowledgements}

Assistance with flow cytometry from Dr. Wu He is appreciated. Assistance with Western blots from Megan Schladetsch is appreciated. DGBP was a generous gift from Dr. David Wiemer at the University of lowa, lowa City, IA. Financial support from the University of Connecticut Vice President for Research (Research Excellence Program) is gratefully acknowledged.

\section{Conflict of interest}

DGBP is covered by a patent that is owned by the University of lowa. A.J.W. owns shares in Terpenoid Therapeutics, Inc., which has licensed the patent.

\section{Publisher's note}

Springer Nature remains neutral with regard to jurisdictional claims in published maps and institutional affiliations.

Received: 6 June 2019 Revised: 9 September 2019 Accepted: 12 September 2019

Published online: 30 September 2019

\section{References}

1. Pui, C. H., Relling, M. V. \& Downing, J. R. Acute lymphoblastic leukemia. N. Engl. J. Med. 350, 1535-1548 (2004).

2. Sanchez-Martin, M. et al. Synergistic antileukemic therapies in NOTCH1induced T-ALL. Proc. Natl Acad. Sci. USA 114, 2006-2011 (2017).

3. Weng, A. P. et al. Activating mutations of NOTCH1 in human T cell acute lymphoblastic leukemia. Science 306, 269-271 (2004).

4. Brzozowa-Zasada, M. et al. Notch and its oncogenic activity in human malignancies. Eur. Surg. 49, 199-209 (2017).

5. Radtke, F. \& Raj, K. The role of Notch in tumorigenesis: oncogene or tumour suppressor? Nat. Rev. Cancer 3, 756-767 (2003).

6. Andersson, E. R., Sandberg, R. \& Lendahl, U. Notch signaling: simplicity in design, versatility in function. Development 138, 3593-3612 (2011).

7. Passaro, D., Quang, C. T. \& Ghysdael, J. Microenvironmental cues for T-cell acute lymphoblastic leukemia development. Immunol. Rev. 271, 156-172 (2016).

8. Olsauskas-Kuprys, R., Zlobin, A. \& Osipo, C. Gamma secretase inhibitors of Notch signaling. Onco Targets Ther. 6, 943-955 (2013).

9. Gordon, W. R. et al. Structural basis for autoinhibition of Notch. Nat. Struct. Mol. Biol. 14, 295-300 (2007).

10. Sanchez-Martin, M. \& Ferrando, A. The NOTCH1-MYC highway toward T-cell acute lymphoblastic leukemia. Blood 129, 1124-1133 (2017).
11. Court, H., Ahearn, I. M., Amoyel, M., Bach, E. A. \& Philips, M. R. Regulation of NOTCH signaling by RAB7 and RAB8 requires carboxyl methylation by ICMT. J. Cell Biol. 216, 4165-4182 (2017).

12. Doi, K. et al. Crucial role of the Rap G protein signal in Notch activation and leukemogenicity of T-cell acute lymphoblastic leukemia. Sci. Rep. 5, 7978 (2015).

13. Shull, L. W., Wiemer, A. J., Hohl, R. J. \& Wiemer, D. F. Synthesis and biological activity of isoprenoid bisphosphonates. Bioorg. Med. Chem. 14, 4130-4136 (2006).

14. Wiemer, A. J., Tong, H., Swanson, K. M. \& Hohl, R. J. Digeranyl bisphosphonate inhibits geranylgeranyl pyrophosphate synthase. Biochem. Biophys. Res. Commun. 353, 921-925 (2007).

15. Maalouf, M. A., Wiemer, A. J., Kuder, C. H., Hohl, R. J. \& Wiemer, D. F. Synthesis of fluorescently tagged isoprenoid bisphosphonates that inhibit protein geranylgeranylation. Bioorg. Med. Chem. 15, 1959-1966 (2007).

16. Wiemer, A. J., Yu, J. S., Lamb, K. M., Hohl, R. J. \& Wiemer, D. F. Mono- and dialkyl isoprenoid bisphosphonates as geranylgeranyl diphosphate synthase inhibitors. Bioorg. Med. Chem. 16, 390-399 (2008).

17. Wiemer, A. J. et al. Pivaloyloxymethyl-modified isoprenoid bisphosphonates display enhanced inhibition of cellular geranylgeranylation. Bioorg. Med. Chem. 16, 3652-3660 (2008)

18. Wiemer, A. J., Wiemer, D. F. \& Hohl, R. J. Geranylgeranyl diphosphate synthase: an emerging therapeutic target. Clin. Pharmacol. Ther. 90, 804-812 (2011).

19. Agabiti, S. S., Li, J. \& Wiemer, A. J. Geranylgeranyl diphosphate synthase inhibition induces apoptosis that is dependent upon GGPP depletion, ERK phosphorylation and caspase activation. Cell Death Dis. 8, e2678 (2017).

20. Wiemer, A. J., Hohl, R. J. \& Wiemer, D. F. The intermediate enzymes of isoprenoid metabolism as anticancer targets. Anticancer Agents Med. Chem. 9, 526-542 (2009)

21. Agabiti, S. S., Liang, Y. \& Wiemer, A. J. Molecular mechanisms linking geranylgeranyl diphosphate synthase to cell survival and proliferation. Mol. Membr. Biol. 33, 1-11 (2016).

22. Haney, S. L. et al. Preclinical investigation of a potent geranylgeranyl diphosphate synthase inhibitor. Invest. New Drugs 36, 810-818 (2018).

23. Lacbay, C. M. et al. Unraveling the prenylation-cancer paradox in multiple myeloma with novel geranylgeranyl pyrophosphate synthase (GGPPS) inhibitors. J. Med. Chem. 61, 6904-6917 (2018).

24. Matthiesen, R. A. et al. Alpha-Methylation enhances the potency of isoprenoid triazole bisphosphonates as geranylgeranyl diphosphate synthase inhibitors. Bioorg. Med. Chem. 26, 376-385 (2018).

25. Zhang, $Y$. et al. Simultaneous site-specific dual protein labeling using protein prenyltransferases. Bioconjug. Chem. 26, 2542-2553 (2015).

26. Jeong, A., Suazo, K. F., Wood, W. G., Distefano, M. D. \& Li, L. Isoprenoids and protein prenylation: implications in the pathogenesis and therapeutic intervention of Alzheimer's disease. Crit. Rev. Biochem. Mol. Biol. 53, 279-310 (2018).

27. Coxon, F. P. et al. Synthesis and characterization of novel phosphonocarboxylate inhibitors of RGGT. Eur. J. Med. Chem. 84, 77-89 (2014).

28. Zhou, X. et al. Triazole-based inhibitors of geranylgeranyltransferase II. Bioorg. Med. Chem. Lett. 23, 764-766 (2013).

29. Zagouras, P., Stifani, S., Blaumueller, C. M., Carcangiu, M. L. \& Artavanis-Tsakonas, S. Alterations in Notch signaling in neoplastic lesions of the human cervix. Proc. Natl Acad. Sci. USA 92, 6414-6418 (1995).

30. Lin, J. J. Monoclonal antibodies against myofibrillar components of rat skeletal muscle decorate the intermediate filaments of cultured cells. Proc. Natl Acad. Sci. USA 78, 2335-2339 (1981).

31. Zhang, T. et al. A genetic cell context-dependent role for ZEB1 in lung cancer Nat. Commun. 7, 12231 (2016).

32. Thompson, K., Rogers, M. J., Coxon, F. P. \& Crockett, J. C. Cytosolic entry of bisphosphonate drugs requires acidification of vesicles after fluid-phase endocytosis. Mol. Pharmacol. 69, 1624-1632 (2006).

33. $\mathrm{Yu}, \mathrm{Z}$. et al. Identification of a transporter complex responsible for the cytosolic entry of nitrogen-containing bisphosphonates. elife 7, e36620 (2018).

34. Rao, S. S. et al. Inhibition of NOTCH signaling by gamma secretase inhibitor engages the RB pathway and elicits cell cycle exit in T-cell acute lymphoblastic leukemia cells. Cancer Res. 69, 3060-3068 (2009).

35. O'Neil, J. et al. FBW7 mutations in leukemic cells mediate NOTCH pathway activation and resistance to gamma-secretase inhibitors. J. Exp. Med. 204, 1813-1824 (2007).

36. Huo, L. et al. Effect of dihydroarteminin combined with siRNA targeting Notch1 on Notch1/c-Myc signaling in T-cell lymphoma cells. Exp. Ther. Med. 15, 3059-3065 (2018) 
37. Zou, J. et al. Notch1 is required for hypoxia-induced proliferation, invasion and chemoresistance of T-cell acute lymphoblastic leukemia cells. J. Hematol. Oncol. 6, 3 (2013).

38. Fox, V. et al. Cell-cell signaling through NOTCH regulates human embryonic stem cell proliferation. Stem Cells 26, 715-723 (2008).

39. Dudakovic, A. et al. Inhibition of geranylgeranyl diphosphate synthase induces apoptosis through multiple mechanisms and displays synergy with inhibition of other isoprenoid biosynthetic enzymes. J. Pharmacol. Exp. Ther. 324 1028-1036 (2008).

40. Weng, A. P. et al. c-Myc is an important direct target of Notch1 in T-cell acute lymphoblastic leukemia/lymphoma. Genes Dev. 20, 2096-2109 (2006).

41. Barila, D. et al. Caspase-dependent cleavage of c-Abl contributes to apoptosis. Mol. Cell. Biol. 23, 2790-2799 (2003).

42. Vermezovic, J. et al. Notch is a direct negative regulator of the DNA-damage response. Nat. Struct. Mol. Biol. 22, 417-424 (2015)

43. De Keersmaecker, $\mathrm{K}$. et al. In vitro validation of gamma-secretase inhibitors alone or in combination with other anti-cancer drugs for the treatment of Tcell acute lymphoblastic leukemia. Haematologica 93, 533-542 (2008).

44. Forbes, S. A. et al. COSMIC: somatic cancer genetics at high-resolution. Nucleic Acids Res. 45, D777-D783 (2017).

45. Dumanchin, C. et al. Presenilins interact with Rab11, a small GTPase involved in the regulation of vesicular transport. Hum. Mol. Genet. 8, 1263-1269 (1999).

46. Morohashi, Y. et al. C-terminal fragment of presenilin is the molecular target of a dipeptidic gamma-secretase-specific inhibitor DAPT ( $\mathrm{N}$-[N-(3,5-difluorophenacetyl)-L-alanyl]-S-phenylglycine t-butyl ester. J. Biol. Chem. 281, 14670-14676 (2006).

47. Carter, J. A. \& Botteman, M. F. Health-economic review of zoledronic acid for the management of skeletal-related events in bone-metastatic prostate cancer. Expert Rev. Pharmacoecon. Outcomes Res. 12, 425-437 (2012).

48. Fournier, P. G. et al. Lowering bone mineral affinity of bisphosphonates as a therapeutic strategy to optimize skeletal tumor growth inhibition in vivo. Cancer Res. 68, 8945-8953 (2008).

49. Lee, J. S. et al. Statins enhance efficacy of venetoclax in blood cancers. Sci. Transl. Med. 10, eaaq1240 (2018).
50. Pandyra, A. et al. Immediate utility of two approved agents to target both the metabolic mevalonate pathway and its restorative feedback loop. Cancer Res. 74, 4772-4782 (2014).

51. Pandyra, A. A. et al. Genome-wide RNAi analysis reveals that simultaneous inhibition of specific mevalonate pathway genes potentiates tumor cell death. Oncotarget 6, 26909-26921 (2015).

52. Brown, M. S. \& Goldstein, J. L. The SREBP pathway: regulation of cholesterol metabolism by proteolysis of a membrane-bound transcription factor. Cell $\mathbf{8 9}$, 331-340 (1997).

53. Dudakovic, A., Tong, H. \& Hohl, R. J. Geranylgeranyl diphosphate depletion inhibits breast cancer cell migration. Invest. New Drugs 29, 912-920 (2011).

54. Sharma, V. M. et al. Notch1 contributes to mouse T-cell leukemia by directly inducing the expression of c-myc. Mol. Cell. Biol. 26, 8022-8031 (2006).

55. Matt, S. \& Hofmann, T. G. The DNA damage-induced cell death response: a roadmap to kill cancer cells. Cell. Mol. Life Sci. 73, 2829-2850 (2016).

56. Korwek, Z. et al. Inhibition of ATM blocks the etoposide-induced DNA damage response and apoptosis of resting human T cells. DNA Repair 11, 864-873 (2012).

57. Wang, J. Y., Naderi, S. \& Chen, T. T. Role of retinoblastoma tumor suppressor protein in DNA damage response. Acta Oncol. 40, 689-695 (2001).

58. Reszka, A. A., Halasy-Nagy, J. \& Rodan, G. A. Nitrogen-bisphosphonates block retinoblastoma phosphorylation and cell growth by inhibiting the cholesterol biosynthetic pathway in a keratinocyte model for esophageal irritation. Mol. Pharmacol. 59, 193-202 (2001).

59. Machuy, N., Rajalingam, K. \& Rudel, T. Requirement of caspase-mediated cleavage of c-Abl during stress-induced apoptosis. Cell Death Differ. 11, 290-300 (2004).

60. Baskaran, R. et al. Ataxia telangiectasia mutant protein activates c-Abl tyrosine kinase in response to ionizing radiation. Nature 387, 516-519 (1997).

61. Shimono, Y., Mukohyama, J., Nakamura, S. \& Minami, H. MicroRNA regulation of human breast cancer stem cells. J. Clin. Med. 5, 1-24 (2015).

62. Xu, Y. F., Hannafon, B. N. \& Ding, W. Q. microRNA regulation of human pancreatic cancer stem cells. Stem Cell Investig. 4, 5 (2017). 